\title{
CASE REPORT \\ Hiccup-like segmental myoclonus in thoracic compressive myelopathy: a case report
}

\author{
C-N Lee, S-H Baek, K-W Park and B-J Kim
}

\begin{abstract}
Study design: A case report.
Objectives: This study discusses a case of spinal segmental myoclonus caused by thoracic myelopathy, mimicking hiccup spasms. Spinal myoclonus caused by thoracic myelopathy is extremely rare. It can be misdiagnosed as chronic intractable hiccups due to similar clinical manifestations.

Setting: Korea University Anam Hospital, Seoul, Republic of Korea.

Methods: A 42-year-old man presented with a history of involuntary jerky movement of the upper abdominal wall muscles that had been continuing for over 3 years. A neurological examination, brain computed tomography and electroencephalogram did not reveal a cause of the symptoms. Electromyography was performed on the abdominal muscles and the findings revealed were compatible with spinal myoclonus. The spinal myoclonus had started in the abdominal muscles, with a spinal magnetic resonance imaging revealing a disc protrusion compressing the anterior spinal cord.

Results: The cause of the spinal myoclonus was determined to be spinal myelopathy due to mild T7 disc protrusion. The patient refused surgical or invasive interventions and was conservatively treated with clonazepam. The symptoms were reported to be less frequent following the treatment.
\end{abstract}

Conclusion: Compressive myelopathy developed from disc protrusion may cause spinal myoclonus mimicking as hiccup spasms. Spinal Cord (2014) 52, S16-S18; doi:10.1038/sc.2014.140

\section{INTRODUCTION}

Myoclonus is defined as a sudden, brief and involuntary muscle contraction, ${ }^{1}$ with the prevalence of myoclonus being 8.6 per 100000 individuals. Spinal myoclonus is rare in contrast to cortical and subcortical myoclonus. Especially, spinal myoclonus due to thoracic compressive myelopathy has not been reported. Herein, we report on a case that presented with hiccup-like myoclonus, which was associated with T6-T7 intervertebral disc protrusion.

\section{CASE REPORT}

A 42-year-old man presented with consistent and jerking abdominal muscle contractions that had persisted for about 3 years and aggravated in the past 2 weeks. Initially, these symptoms were diagnosed as persistent hiccups, and no specific treatment was given. None of his family members had shown similar symptoms. The patient noticed that the contraction frequency increased when he was in a supine position or during conditions of emotional stress, and that the symptoms decreased while walking and standing. During sleep, the frequency of contractions decreased but did not stop completely. When tested, the symptoms did not respond to auditory or tactile stimulations. A neurological examination was performed, which revealed high cortical function and normal cranial nerve function. The patient's motor ability and general sensory modality, deep tendon reflexes and the examination of autonomic and cerebellar systems were within the normal range. There was no evidence of pathologic reflexes, and the routine biochemical laboratory examinations were within the normal range. The patient underwent a brain computed tomography and electroencephalogram for a differential diagnosis of cortical and subcortical myoclonus, and they revealed no abnormal findings. Simulations of somatosensory-evoked potentials on the posterior tibial and median nerves did not reveal any abnormal findings. However, a needle electromyography (EMG) showed irregular burst waves of muscle activity, with a rate of $0.2-0.5 \mathrm{~Hz}$ and a duration of $1000 \mathrm{~ms}$, in the muscles of the right upper abdominal wall (innervated by the T7-T10 spinal roots). The test indicated that burst waves were propagated to the right lower and left upper muscles of the abdomen. Each potential was evoked in the right lower, left upper and left lower parts, in sequence with similar intervals of $\sim 15-20 \mathrm{~ms}$ (velocity was $4-5 \mathrm{~m} \mathrm{~s}^{-1}$ with a $1000-\mathrm{ms}$ duration) (Figure 1). A spinal magnetic resonance imaging revealed T6-T7 intervertebral disc protrusion to the right side and high signal intensity of the T7 right anterior horn segment. As the medial region of the anterior horn contained neurons that innervated the flexor muscles of the trunk (Figure 2), the patient was diagnosed with spinal myoclonus caused by thoracic cord compression. The patient refused any surgical or interventional treatment and was conservatively treated with clonazepam $\left(1.5 \mathrm{mg} \mathrm{day}^{-1}\right)$. The symptoms became less frequent with this treatment about 2 days later but did not disappear. The patient felt more comfortable with the use of this medicine at same dose for about 2 months, and then stopped visiting our clinic himself.

A written informed consent was exempted by our IRB for this case report. 
a

\section{b}

Right upper Rectus Abdominalis

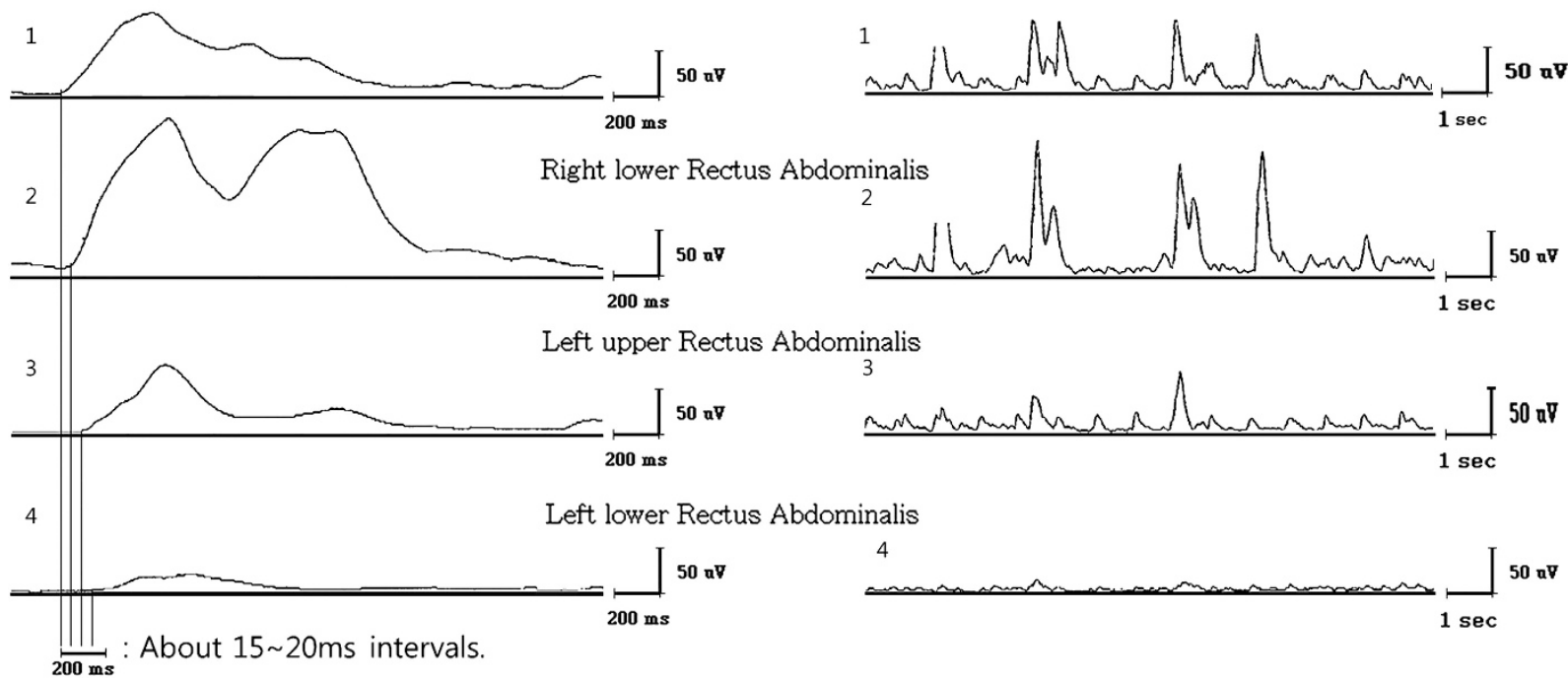

Figure 1 Four-channel needle EMG. (a) EMG shows muscle contraction and a potential with long duration is evoked in the right upper rectus abdominis muscle. (b) Four-channel needle EMG shows irregular bursts of muscle contractions.

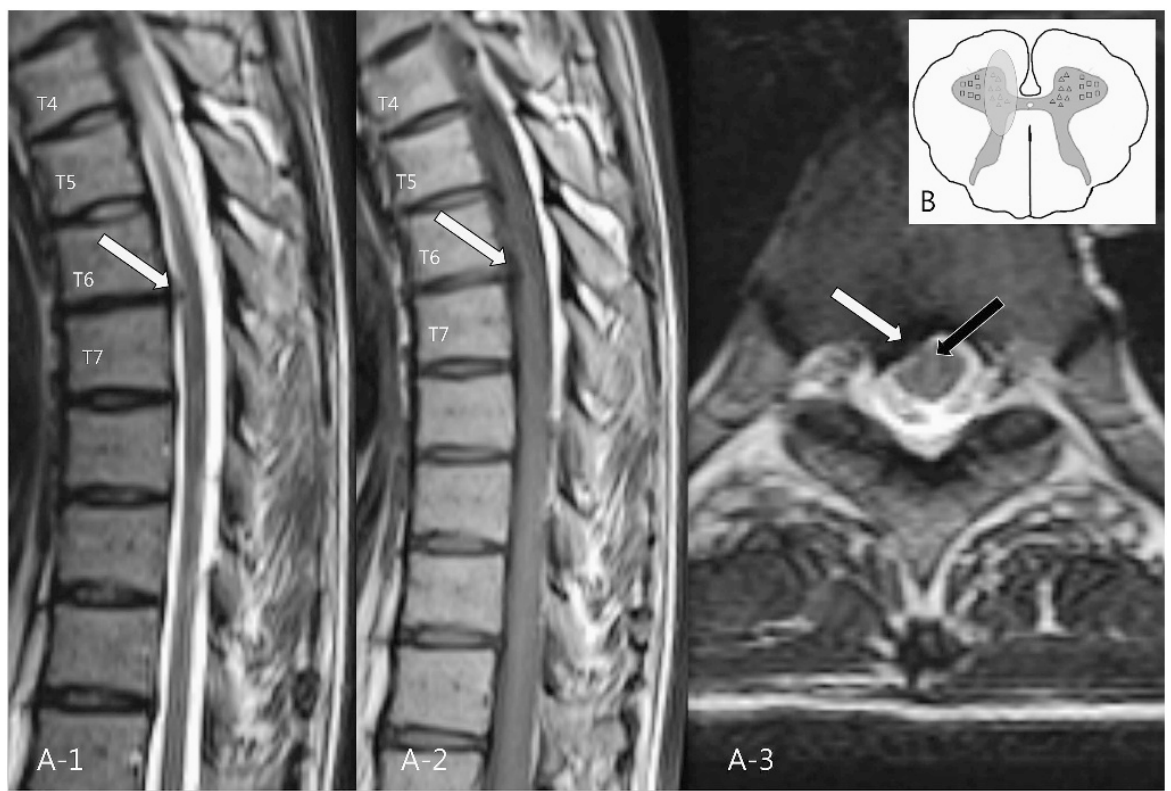

Figure 2 (A-1, A-2) Sagittal T2- and T1-weighted magnetic resonance imaging (MRI) at the T6-T7 levels. Disc protrusion (white arrow) and thoracic cord compression are revealed. (A-3) Axial T2-weighted MRI at the T6-T7 level. The image indicates disc protrusion (white arrow) and hyperintensity in the right medial anterior horn cells (black arrow). (B) Schematic diagram of a lesion in a spinal segment.

\section{DISCUSSION}

Abdominal myoclonus is differentiated from hiccup spasms. Hiccups usually present with noise created by air, which is expelled from the lungs with sudden diaphragmatic contraction. ${ }^{1}$ Our patient had experienced consistent symptoms for nearly 3 years, and as the symptoms persisted during sleep and distress, psychogenic myoclonus was eliminated. The pathology of spinal myoclonus is consistent with a primary abnormality in the spinal cord. Previous studies have suggested mechanisms that explain the causes of spinal segmental myoclonus. These mechanisms include abnormal neural hyperactivity of the anterior horn cells due to direct cellular excitation by a tumor, infarction or inflammation; neural activation due to removal of an inhibition; ${ }^{2}$ aberrant local axons or cellular level re-excitations, especially in cases of hemifacial spasms; and finally, a loss of inhibition from suprasegmental descending pathways. ${ }^{3,4}$ One previously reported study suggested excitability of anterior horn cells as a pathogenesis of spinal myoclonus based on a case of myoclonus that showed 
vacuolar degeneration of anterior horn cells from chronic compression. ${ }^{5}$

This study proposes that a lesion in the T7 segment, developed due to anterior compression, caused the spinal myoclonus and presented as abdominal twitching similar to chronic intractable hiccups. This proposal is based on a consideration of the anterior horn topography, which includes neurons in the medial area of the anterior horn (triangle) that innervate axial muscles, in addition to neurons in the lateral area (square) that innervate distal limb muscles (Figure 2B). The patient needed surgical intervention to resolve the symptoms; however, he refused to undergo invasive treatment and thus was treated conservatively.

Cases with similar presentation may be extremely rare. However, chronic hiccups should be differentiated from the spinal segmental myoclonus that does not have noise created by air and persists during sleep. In addition, EMG showing burst waves propagated to the muscles innervated by lower spinal segments may help to diagnose spinal myoclonus.

\section{CONFLICT OF INTEREST}

The authors declare no conflict of interest.

1 Fahn S, Jankovic J, Hallett M. Principles and Practice of Movement Disorders, 2nd edn. Elsevier Saunders: Amsterdam, 2011, pp 450-454.

2 Swanson PD, Luttrell CN, Magladery JW. Myoclonus-a report of 67 cases and review of the literature. Medicine (Baltimore) 1962; 41: 339-356.

3 Roth G, Magistris MR, Pinelli P, Rilliet B. Cryptogenic hemifacial spasm. A neurophysiological study. Electromyogr Clin Neurophysiol 1990; 30: 361-370.

4 Campos CR, Limongi JC, Machado FC, Brotto MW. A case of primary spinal myoclonus: clinical presentation and possible mechanisms involved. Arq Neuropsiquiatr 2003; 61: 112-114.

5 Shivapour E, Teasdall RD. Spinal myoclonus with vacuolar degeneration of anterior horn cells. Arch Neurol 1980; 37: 451-453. 